Kelkel, Mareike; Peschel, Markus

\title{
Förderung der beruflichen Handlungsfähigkeit von Studierenden im Sachunterricht durch das Gofex_Projektpraktikum
}

Tänzer, Sandra [Hrsg.]; Godau, Marc [Hrsg.]; Berger, Marcus [Hrsg.]; Mannhaupt, Gerd [Hrsg.]: Perspektiven auf Hochschullernwerkstätten. Wechselspiele zwischen Individuum, Gemeinschaft, Ding und Raum. Bad Heilbrunn : Verlag Julius Klinkhardt 2019, S. 157-167. - (Lernen und Studieren in Lernwerkstätten)

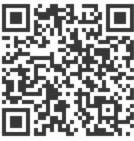

Quellenangabe/ Reference:

Kelkel, Mareike; Peschel, Markus: Förderung der beruflichen Handlungsfähigkeit von Studierenden im Sachunterricht durch das Gofex_Projektpraktikum - In: Tänzer, Sandra [Hrsg.]; Godau, Marc [Hrsg.]; Berger, Marcus [Hrsg.]; Mannhaupt, Gerd [Hrsg.]: Perspektiven auf Hochschullernwerkstätten. Wechselspiele zwischen Individuum, Gemeinschaft, Ding und Raum. Bad Heilbrunn : Verlag Julius Klinkhardt 2019, S. 157-167 - URN: urn:nbn:de:0111-pedocs-201968 - DOI: 10.25656/01:20196

https://nbn-resolving.org/urn:nbn:de:0111-pedocs-201968

https://doi.org/10.25656/01:20196

in Kooperation mit / in cooperation with:

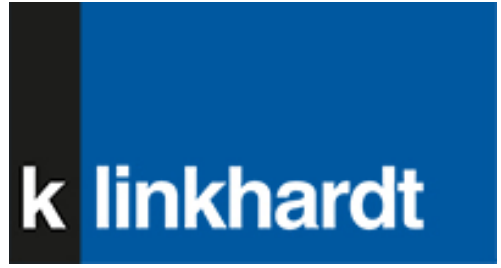

http://www.klinkhardt.de

\section{Nutzungsbedingungen}

Dieses Dokument steht unter folgender Creative Commons-Lizenz: http://creativecommons.org/licenses/by-nc-sa/4.0/deed.de - Sie dürfen das Werk bzw. den Inhalt unter folgenden Bedingungen vervielfältigen, verbreiten und öffentlich zugänglich machen sowie Abwandlungen und Bearbeitungen des Werkes bzw. Inhaltes anfertigen: Sie müssen den Namen des Autors/Rechteinhabers in der von ihm festgelegten Weise nennen. Dieses Werk bzw. der Inhalt darf nicht für kommerzielle Žwecke verwendet werden. Die neu entstandenen Werke bzw. Inhalte dürfen nur unter Verwendung von Lizenzbedingungen weitergegeben werden, die mit denen dieses Lizenzvertrages identisch oder vergleichbar sind.

Mit der Verwendung dieses Dokuments erkennen Sie die Nutzungsbedingungen an.

\section{Terms of use}

This document is published under following Creative Commons-License: http://creativecommons.org/licenses/by-nc-sa/4.0/deed.en - You may copy, distribute and transmit, adapt or exhibit the work in the public and alter, transform or change this work as long as you attribute the work in the manner specified by the author or licensor. You are not allowed to make commercial use of the work. If you alter, transform, or change this work in any way, you may distribute the resulting work only under this or a comparable license.

By using this particular document, you accept the above-stated conditions of use.

\section{Kontakt / Contact:}

\section{DeDOCS}

DIPF | Leibniz-Institut für Bildungsforschung und Bildungsinformation Informationszentrum (IZ) Bildung

E-Mail: pedocs@dipf.de

Internet: www.pedocs.de

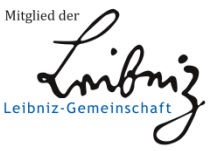




\section{Mareike Kelkel und Markus Peschel}

\section{Förderung der beruflichen Handlungsfähigkeit von Studierenden im Sachunterricht durch das GOFEX_Projektpraktikum ${ }^{1}$}

\section{Zusammenfassung}

Dieser konzeptionelle Beitrag beschreibt Idee und Umsetzung des GOFEX_Projektpraktikums, welches als praxisnahes Element in der Sachunterrichtsausbildung angehender Grundschullehrkräfte auf die Förderung der beruflichen Handlungsfähigkeit der Studierenden zielt. Im Fokus steht hierbei die Steigerung der Reflexionsfähigkeit des eigenen Handelns in der Rolle als Lernbegleitung beim Experimentieren im Sachunterricht. Ergebnisse einer ersten Evaluation und Erfahrungen von Studierenden unterstützen die Konzeption der Lehrveranstaltung: einerseits deuten die Antworten der Studierenden auf eine Reflexionssteigerung hin. Andererseits nehmen die Studierenden das Einnehmen der unterschiedlichen Rollen im GOFEX_Projektpraktikum als Lerngelegenheit wahr.

\section{Sachunterrichtsausbildung an der Universität des Saarlandes}

Das Studium für das Lehramt an der Universität des Saarlandes (UdS) orientiert sich an der Entwicklung grundlegender beruflicher Kompetenzen für Unterricht und Erziehung [...] (vgl. Studienordnung UdS 2018, 327). Für künftige saarländische Grundschullehrkräfte bezieht sich das berufliche Handeln insbesondere auf das Handlungsfeld Unterricht, dessen Durchführung die Kernaufgabe von Grundschullehrkräften ist. Das Handlungsfeld Unterricht umfasst dabei die Planung, Gestaltung, Durchführung, Evaluation und Reflexion von fachlichen und überfachlichen Lehr-Lern-Prozessen, die in den Studienzielen für das Lehramt Primarstufe an der Universität des Saarlandes beschrieben werden. Insbesondere die Fähigkeit zur Reflexion ist als generische Kompetenz im Qualifikationsrahmen für deutsche Hochschulabschlüsse beschrieben und gehört damit zum Qua-

1 Das dargestellte Projekt wird im Rahmen der vom BMBF geförderten „Qualitätsoffensive Lehrerbildung" durchgeführt und ist Bestandteil des saarländischen Verbundprojektes SaLUt (Optimierung der saarländischen Lehrerinnenausbildung: Förderung des Umgangs mit Heterogenität und Individualisierung im Unterricht). Förderkennzeichen: 01JA1606A. 
lifikationsprofil aller Absolvent*innen, wobei die Reflexion durch wissenschaftliche/erkenntnistheoretische Prinzipien strukturiert ist (KMK 2017, 3f.). Auf der Qualifikationsebene Master ${ }^{2}$ wird zu Reflexivität u.a. formuliert:

Absolvent*innen [...]

- begründen das eigene berufliche Handeln mit theoretischem und methodischem Wissen und reflektieren es hinsichtlich alternativer Entwürfe;

- schätzen die eigenen Fähigkeiten ein, nutzen sachbezogene Gestaltungs- und Entscheidungsfreiheiten autonom und entwickeln diese unter Anleitung weiter;

- erkennen situationsadäquat und situationsübergreifend Rahmenbedingungen beruflichen Handelns und reflektieren Entscheidungen verantwortungsethisch;

- reflektieren kritisch ihr berufliches Handeln in Bezug auf gesellschaftliche Erwartungen und Folgen und entwickeln ihr berufliches Handeln weiter (KMK 2017, 7f.).

In der Ausbildung zukünftiger Sachunterrichts-Lehrkräfte an der Universität des Saarlandes wird in verschiedenen Modulen die Fähigkeit zu reflexivem Handeln gefördert. Zu beachten ist dabei aus curricularer Sicht, dass die genannten Kernaufgaben (Planung, Gestaltung, Durchführung, Evaluation und Reflexion) nicht unabhängig voneinander erlernbar und nur gemeinsam wirksam für einen erfolgreichen zeitgemäßen Unterricht sind (vgl. FSA 2018, 486). Dies bedeutet, dass Reflexionsfähigkeit nicht isoliert gefördert werden sollte, sondern in einem (direkten/indirekten) Bezug zum Unterricht stehen muss. Zur eigenen Kompetenzentwicklung sollen Studierende zudem die Möglichkeit erhalten, im Studium grundlegende Erfahrungen in der Verknüpfung von Theorie und Praxis in beruflichen Aufgabenfeldern zu gewinnen (vgl. Studienordnung UdS 2018, 327). Vor diesem Hintergrund wurde als neues Modulelement das Gofex_Projektpraktikum (Gofex_PP) als weiteres Praxisformat im Studiengang Lehramt Primarstufe entwickelt und mit weiteren Modulen im GoFex sowie dem bestehenden Schulpraktikum konzeptionell verknüpft.

\section{Reflexion im und durch das GOFEX_Projektpraktikum}

\subsection{GoFEX_Projektpraktikum - Allgemeines Konzept}

Das Grundschullabor für Offenes Experimentieren (Gofex) ist als Hochschullernwerkstatt bereits fest in der Sachunterrichtsausbildung im Studiengang Lehramt Primarstufe der Universität des Saarlandes verankert und zwei Teilmodule (Modul 3a und 3b) fokussieren die Entwicklung von (offenen) Experimentier-

2 Der Abschluss Master of Education berechtigt zur Aufnahme des Referendariats und ist mit dem 1. Staatsexamen der UdS gleichzusetzen. 
situationen. Das Gofex ist zudem Mitglied des Verbundes der Lernwerkstätten (VdL), der sich im Rahmen des Projektes SaLUt der „Qualitätsoffensive Lehrerbildung" (BMBF) in die Lehramtsstudiengänge integriert, mit dem Ziel, das Lehramtsstudium praxisorientierter zu gestalten und die Studierenden durch neue Angebote gezielt auf den Umgang mit Heterogenität und Individualisierung im Unterricht vorzubereiten (https://www.lernwerkstatt.saarland). Dabei soll der $\mathrm{VdL}$ als strukturell vernetzendes Moment insbesondere die wissenschaftsgestützte Praxisorientierung ${ }^{3}$ verbessern.

Das im VdL bzw. SaLUt entwickelte und im Studiengang Lehramt Primarstufe angesiedelte Modulelement Gofex_PP gibt den Studierenden insbesondere die Möglichkeit, ihre Rolle als Lernbegleitung in der Praxis aktiv zu erproben und in der Gesamtheit auf das Studienziel „Planung, Gestaltung, Durchführung, Evaluation und Reflexion von Lernprozessen“ hinzuwirken. Das GofEx_PP ${ }^{4}$ gliedert sich dabei in zwei Teile:

1. einen praktischen Teil, in dem die Studierenden Schulklassen im Gofex oder an Kooperationsschulen begleiten (mind. 2 SWS), und ein

2. theoretisch angelegtes Begleitseminar, in dem Praxis theoriegeleitet interpretiert wird und v.a. die (gemeinsame) Evaluation und Reflexion der im Praxisteil gemachten Erfahrungen und Beobachtungen im Vordergrund stehen (2 SWS).

Im praktischen Teil werden die Studierenden angehalten, sich eigenständig mit dem naturwissenschaftlichen Experimentieren, der sukzessiven Öffnung und Individualisierung von Lernsituationen auseinanderzusetzen (vgl. Peschel 2009, 2016) und entdeckendes Lernen im Gofex und in den Grundschulen zu ermöglichen. Die Studierenden konnten im Vorfeld des Projektpraktikums bereits in ein bis zwei aufeinander aufbauenden Gofex-Seminaren Erfahrung in der Planung und Gestaltung von Lernumgebungen sammeln und sich mit dem Öffnungskonzept, welches sich an der stufenweisen Öffnung von Grundschulunterricht nach Falko Peschel (2012) orientiert, vertraut machen. Das Gofex_PP baut auf diesen Vorkenntnissen (Konzeption, Öffnungsstufen, Kriterien guter Aufgaben, Wege der gemeinsamen Erkenntnis) auf und erweitert die in den vorangegangenen Seminaren theoretisch entwickelten/selbst erstellten Experimentierumgebungen um die Durchführung, Evaluation und Reflexion (vgl. Kelkel \& Peschel 2018, 25ff.).

3 Im VdL findet Forschung u.a. im Rahmen wissenschaftlicher Abschlussarbeiten statt. Die entwickelten Lernwerkstattangebote werden kontinuierlich evaluiert und auf der Basis der erhaltenen Ergebnisse weiterentwickelt und optimiert.

4 Die Studierenden können wählen, ob sie neben dem Begleitseminar (2 ETCS) ein kleines, mittleres oder großes Projektpraktikum belegen und entsprechend 2, 4 oder 6 ECTS dafür erhalten. Alle Studierenden (2, 4 oder 6 ECTS) reflektieren ihren Lernprozess in einer schriftlichen Ausarbeitung. Beim mittleren und großen Projektpraktikum ist zusätzlich die Überarbeitung von Experimentiereinheiten als Prüfungsleistung enthalten und weitet die ECTS entsprechend aus. 
Die entwickelten Experimentierumgebungen sollen während des Gofex-Projektpraktikums theoriegeleitet und erfahrungsbasiert reflektiert sowie optimiert werden, wobei einerseits die im Begleitseminar geteilten bzw. reflektierten Eindrücke beim Einsatz mit Schüler*innen, andererseits die Rückmeldungen der Mitstudierenden, die die Experimentiereinheiten im Seminar praktisch erproben, einfließen.

\subsection{Förderung der Reflexivität durch das Einnehmen verschiedener Rollen}

Darüber hinaus dient das Begleitseminar dazu, auf das Konzept von Lernwerkstatt(arbeit) und auf die Rolle der Lernbegleitung beim Experimentieren einzugehen. Um eine tiefergehende Reflexion des eigenen Handelns zu ermöglichen, nehmen die Studierenden im GofEx_PP während der gesamten Veranstaltung immer wieder unterschiedliche Rollen/Perspektiven ein, aus denen heraus sie analysieren und reflektieren sollen: Lernbegleitende, Beobachtende und Experimentierende.

1. Lernbegleitende: Ziel der Rolle als Lernbegleiter*in ist es, das eigene Verhalten in Lehr-Lern-Situationen bewusst wahrzunehmen und zu reflektieren, wie sich eine „gute Lernbegleitung" - hier mit Bezug zum Offenen Experimentieren - darstellt, und wie das eigene Verhalten in Bezug dazu steht. Die Studierenden sollen sich hierbei an den von Hagstedt (2014) formulierten Prinzipien orientieren, die das Handeln der Lernbegleitenden leiten: (1) informelles Lernarrangement, (2) dialogisches Mitforschen und (3) lernbiographische Spurensuche.

„Die Lernbegleitung ist grundsätzlich verantwortlich für den Entwurf einer Lernumgebung" (Hagstedt 2014, 227). Diese Aufgabe übernimmt im GofEx_PP die „Hauptlernbegleitung“, die den Schüler*innentag im Gofex plant und hauptverantwortlich durchführt (statt Mitarbeitenden des Lehrstuhls können die Studierenden GofEx_PP diese Rolle einnehmen und führen den Tag eigenverantwortlich durch). Darüber hinaus können die Studierenden als „Nebenlernbegleiter*innen" die Hauptlernbegleitenden unterstützen und als dialogische Mitforschende agieren. Dabei versuchen die Nebenlernbegleiter*innen „durch leises Mit-Denken und dosiertes Zurück-Fragen“ (ebd., 228) herauszufinden, wie die Lernenden (Schüler*innen) denken, um die individuellen Lernwege der Kinder nachzuverfolgen bzw. zu rekonstruieren. Beides dient letztendlich dazu, den Kindern individuelle Zugänge und Lern(um)wege zu ermöglichen (vgl. VeLW 2009; Wedekind \& Schmude 2017). Die Gestaltung und Durchführung der Lehr-Lerneinheit steht im Fokus dieser Rolle - und zwar einerseits in Hinblick auf die eigene Interaktion mit den Lernenden und im Falle der Hauptlernbegleitung im Hinblick einer Evaluation der eingesetzten Lernumgebung.

2. Beobachtende: Ziel der Rolle als Beobachter*in ist es, das Verhalten anderer Lernbegleitenden in diesen Lehr-Lern-Situationen bewusst wahrzunehmen und zu reflektieren, wie das jeweilige Verhalten der Mitstudierenden (s. Rolle 1: 
Lernbegleitung) als „gute Lernbegleitung“ in Bezug zur theoretisch erarbeiteten Position steht. Sie analysieren - teilweise auf mehrere Beobachtende verteilt in dieser Rolle die Interaktionen zwischen (a) den Schüler*innen, (b) zwischen Schüler*innen und den anwesenden Lernbegleitenden bzw. Lehrkräften, sowie (c) zwischen Lehrperson und Lernbegleitendem und setzen die Interaktionen in Bezug zum Handeln der Schüler*innen bzw. dem Lernprozess. Um die gewonnenen Einsichten für Mitstudierende zu verdeutlichen sowie die Aktionen später zu ko-reflektieren, erstellen Beobachtende Videovignetten (mit Hilfe von Tablets oder Digitalkameras aufgenommene kurze Videosequenzen) und präsentieren diese sowie die anschließende Analyse im Seminar.

3. Experimentierende: Ziel der Rolle als Experimentator*in ist es, die (selbst) entworfenen Experimentiereinheiten im Vorfeld aktiv zu testen und im Sinne einer Unterrichtsplanung zu überarbeiten. Aus fachwissenschaftlicher Sicht sollen die Studierenden die Vielfalt an Beobachtungsmöglichkeiten des eingesetzten Experiments erkennen und prüfen, welche Schlüsse diese Beobachtungen zulassen. Aus fachdidaktischer Sicht sollen die Studierenden prüfen, ob es sich um gute Aufgaben (vgl. Peschel 2016) handelt, in denen individuelle methodische Vorgehensweisen ermöglicht werden, verschiedene Lernziele zugelassen sind, Beobachtungen nicht vorweggenommen werden und ob die Aufgaben an die Zielgruppe angepasst sind. Die erarbeiteten Stationen werden beispielsweise in Bezug auf den fachlichen Inhalt/Auswahl der Stationen, sprachlichen Aufbau, Text-Bild-Verhältnis diskutiert und die Ergebnisse können in eine Überarbeitung vor Einsatz in Rolle 1 oder 2 (s.o.) einfließen. Auch die Öffnungsstufe (vgl. Peschel 2009) wird thematisiert und geprüft, inwiefern eine höhere Öffnung der Aufgaben möglich und sinnvoll ist.

\subsection{Reflexion zur Förderung der beruflichen Handlungsfähigkeit}

Die Evaluation und Reflexion im begleitenden Seminar erfolgen in drei Schritten: 1. individuelle Erarbeitung (Konstruktion),

2. Austausch in der Gruppe (Ko-Konstruktion einer gemeinsamen Lösung) und

3. Vorstellen im Plenum (Ko-Konstruktion durch die Zuhörenden).

Konstruktion: Jede/r Studierende wird zunächst selbst aktiv, indem er/sie eine der oben aufgeführten Rollen/Perspektiven einnimmt und ggf. ein Lerntagebuch führt ${ }^{5}$ und die Erfahrungen - zunächst für sich selbst - kritisch analysiert. Das aktive Handeln als Lernbegleitung oder Experimentator*in und die (selbst)kritische Betrachtung der Handlung ist Voraussetzung für die im nächsten Schritt erfolgende ...

5 Das Führen eines Lerntagebuches ist freiwillig, da eine entsprechende Vorgabe unserer Meinung nach dem Sinn eines Lerntagebuches widerspräche. 
Ko-Konstruktion: Die Studierenden tauschen im Seminar ihre Erfahrungen/ Beobachtungen aus und diskutieren in der Gruppe die vorab selbst reflektierten Ansichten und Perspektiven. Hierbei profitieren die Studierenden von der Perspektivenänderung, insbesondere von der Beobachter-Rolle sowie der Gegenüberstellung von Eigen- und Fremdwahrnehmungen. Durch den Einsatz von Videovignetten und die geschilderten Beobachtungen zeigen sich immer wieder Differenzen in der Eigen- und Fremdwahrnehmung, die die Studierenden dahingehend sensibilisieren, das eigene Verhalten in Lehr-Lern-Situationen noch intensiver bzw. kritischer zu reflektieren. Es entsteht eine als „soziale Interdependenz" bezeichnete positive gegenseitige Abhängigkeit der Lernenden: Die Zielerreichung (z.B. das Lösen eines aufgetretenen Problems) wird gemeinsam, mithilfe der Erfahrungen der Mitlernenden erreicht (vgl. Martschinke \& Kopp 2015).

Dabei sollten sich nach Möglichkeit alle Gruppenmitglieder des GofEx_PP bzw. des Lehr-Lern-Prozesses beim Experimentieren gleichberechtigt beteiligen (ebd.), wobei „die Verantwortlichkeit sowohl beim Einzelnen als auch bei der Gruppe“ (ebd., 424) liegt. Die Interaktionsform der gemeinsamen Reflexion wurde gewählt, da Peer-to-peer-Interaktionen laut Piaget einen positiveren Effekt auf das Lernen haben als die Interaktion mit Personen größerer Autorität und Kompetenz, was eine Gefahr der "oberflächlichen Übernahme der Expertenmeinung" (Piaget in Renkl 2007) reduziert. Die Seminarleitung regt durch gezielte Fragen gegebenenfalls weitergehende Reflexionen in Bezug auf spezifische Aspekte an.

\section{Ergebnisse einer ersten Evaluation und Erfahrungen von Studierenden}

Das GofEX_PP wurde erstmals im Wintersemester 2016/17 durchgeführt. Im Sommersemester 2018 fand nun das vierte GoFEx-Projektpraktikum statt, welches erstmals in Kooperation mit den Bildungswissenschaften der Universität des Saarlandes (Prof. Dr. Franziska Perels, Dr. Laura Dörrenbächer-Ulrich) mittels einer Fragebogenerhebung evaluiert wurde. Ziel der Evaluation ist u.a. die Untersuchung, inwieweit das auf Evaluation und Reflexion angelegte Begleitseminar und das Einnehmen der unterschiedlichen Rollen als Lerngelegenheit, Chance oder Belastung wahrgenommen werden. Die Studierenden wurden gebeten, verschiedenen Items auf einer vier-stufigen Skala $(1=$ stimmt gar nicht, $4=$ stimmt genau) einzuschätzen. Zudem wurden Portfolios geführt bzw. mit Lerntagebuchauszügen ergänzt. 
Tab. 1a: Auswertung (absolut) Fragebogen (n=9) (Fortsetzung Tabelle 1b)

\begin{tabular}{|c|c|c|c|c|}
\hline Frage & $\begin{array}{l}\text { Nein, } \\
\text { stimmt } \\
\text { gar } \\
\text { nicht }\end{array}$ & $\begin{array}{l}\text { Nein, } \\
\text { stimmt } \\
\text { eher } \\
\text { nicht }\end{array}$ & $\begin{array}{l}\text { Ja, } \\
\text { stimmt } \\
\text { eher }\end{array}$ & $\begin{array}{l}\text { Ja, } \\
\text { stimmt } \\
\text { genau }\end{array}$ \\
\hline \multicolumn{5}{|l|}{ Allgemein } \\
\hline Ich habe das Gefühl in der Veranstaltung Wichtiges gelernt zu haben. & 1 & 2 & 4 & 2 \\
\hline $\begin{array}{l}\text { Ich habe neue Erkenntnisse für meine spätere Tätigkeit als Lehrkraft } \\
\text { erworben. }\end{array}$ & & 3 & 3 & 3 \\
\hline Die Seminarinhalte sind für mich im späteren Arbeitsalltag nutzbar. & & 1 & 6 & 2 \\
\hline Durch die Praxisphasen habe ich viel gelernt. & & 1 & 3 & 5 \\
\hline \multicolumn{5}{|l|}{ Begleitseminar } \\
\hline $\begin{array}{l}\text { Die gemeinsame Reflexion der Erfahrungen mit den SuS hat mir } \\
\text { geholfen. }\end{array}$ & & 1 & 4 & 4 \\
\hline Ich habe ein Lerntagebuch geführt. & 1 & 5 & 2 & 1 \\
\hline $\begin{array}{l}\text { Meine Aufzeichnungen im Lerntagebuch haben meine Selbstreflexion } \\
\text { positiv beeinflusst. }\end{array}$ & & 4 & 4 & 1 \\
\hline $\begin{array}{l}\text { Durch die Reflexion der Praxistage sind mir die Chancen von offenem } \\
\text { Unterricht bewusst geworden. }\end{array}$ & & 1 & 4 & 4 \\
\hline $\begin{array}{l}\text { Durch die Reflexion der Praxistage sind mir die Grenzen von offenem } \\
\text { Unterricht bewusst geworden. }\end{array}$ & & 1 & 5 & 3 \\
\hline $\begin{array}{l}\text { Der Austausch mit anderen hat meine Selbstreflexion positiv } \\
\text { Beeinflusst. }\end{array}$ & & & 6 & 3 \\
\hline $\begin{array}{l}\text { Das eigene Reflektieren der für das Seminar entwickelten } \\
\text { Lernumgebung war hilfreich für meinen zukünftigen Unterricht. }\end{array}$ & & 1 & 4 & 4 \\
\hline $\begin{array}{l}\text { Das eigene Diskutieren (hinsichtlich Aufgabenqualität und Offenheit) } \\
\text { der für das Seminar entwickelten Lernumgebung war hilfreich für } \\
\text { meinen zukünftigen Unterricht. }\end{array}$ & & 1 & 4 & 4 \\
\hline Das GOFEX_PP hat meine Selbstreflexion gefördert. & & 1 & 6 & 2 \\
\hline Durch das GOFEX_PP reflektiere ich mein Handeln mehr. & & 3 & 5 & 1 \\
\hline Durch das GOFEX_PP reflektiere ich mein Handeln kritischer. & & 3 & 4 & 2 \\
\hline $\begin{array}{l}\text { Durch das GOFEX_PP handle ich bewusster und werde zur } \\
\text { Eigenreflexion angeregt. }\end{array}$ & & 3 & 4 & 2 \\
\hline
\end{tabular}


Tab. 1b: Auswertung (absolut) Fragebogen ( $n=9$ ) (Fortsetzung)

\begin{tabular}{|c|c|c|c|}
\hline \multicolumn{4}{|l|}{ Rollen/Perspektiven } \\
\hline $\begin{array}{l}\text { Durch das Beobachten anderer Lernbegleiter*innen habe ich wichtige } \\
\text { Erkenntnisse für meine Rolle als Lernbegleitung gewonnen. }\end{array}$ & & 6 & 3 \\
\hline $\begin{array}{l}\text { Meine Erfahrungen in der Beobachter*innen- Rolle haben mir selbst } \\
\text { geholfen. }\end{array}$ & 1 & 6 & 2 \\
\hline $\begin{array}{l}\text { Meine Erfahrungen in der Beobachter*innen- Rolle haben den } \\
\text { Mitstudierenden geholfen. }\end{array}$ & & 8 & 1 \\
\hline Das Einnehmen der Lernbegleiter*innen- Rolle hat mir geholfen. & 1 & 4 & 4 \\
\hline $\begin{array}{l}\text { Das Einnehmen der Experimentator*innen- Rolle war hilfreich für die } \\
\text { Überarbeitung meiner Experimentiereinheiten. }\end{array}$ & & 6 & 3 \\
\hline $\begin{array}{l}\text { Das Einnehmen der Experimentator*innen-Rolle war hilfreich für die } \\
\text { Erstellung guter Aufgaben zum Experimentieren. }\end{array}$ & 1 & 6 & 2 \\
\hline $\begin{array}{l}\text { Das eigene Ausprobieren (Experimentieren) der für das Seminar } \\
\text { entwickelten Lernumgebung war hilfreich für meinen zukünftigen } \\
\text { Unterricht. }\end{array}$ & 2 & 4 & 3 \\
\hline
\end{tabular}

Die Auswertung der Ergebnisse der Teilnehmenden $(n=9)^{6}$ zeigt, dass die Praxisphase, das Begleitseminar und die Rollen-/ Perspektivenwechsel mehrheitlich als Lerngelegenheit/Chance wahrgenommen wurden.

Zudem wurden die Studierenden angeregt, ihre Rollen zu reflektieren und dies zu verschriftlichen. Katharina ${ }^{7}$ beschrieb ihre Erfahrungen in der Rolle der Beobachterin in Bezug auf eine Lernbegleitung folgendermaßen:

„Das Beobachten der Lernbegleitung durch andere hat mich selbst in meiner Rolle als Lernbegleitung beeinflusst: als positiv erachtete Verhaltensweisen wurden nachgeahmt (z.B. Anregungen ohne verbale Kommunikation); als negativ erachtete Verhaltensweisen wurden bewusst vermieden (als negativ wurde vor allem das zu starke Eingreifen von Lernbegleitern in das Experimentieren der Kinder wahrgenommen). "

In ihrem Leistungsnachweis erläutert sie hierzu ein konkretes Beispiel: „Besonders interessant fand ich auch, dass es manchmal gar keine Worte benötigt, um die Schüler zum Weiterexperimentieren anzuregen. Dies hatte ich zuvor in der Rolle des Beobachters bei einer Lernbegleitung gesehen und es ergab sich eine Situation, in der ich dies umsetzen konnte. So beobachtete ich eine kleine Gruppe von Schülern an einer Station, an der mit Wasser und Knete experimentiert werden sollte. Die Schüler nahmen jeweils etwas Knete in die Hand, warfen sie in die Schüssel mit Wasser und bestätigten sich ge-

6 Aufgrund der Intensität wird das Seminar (zunächst) in geringer Gruppengröße von bis zu 12 Studierenden durchgeführt. Da die Auswertung aus der ersten (externen) Evaluation resultiert, ist die Fallzahl überschaubar und in Absolutwerten angegeben.

7 Die Namen aller Studierenden wurden geändert. 
genseitig "Knete schwimmt nicht“. Danach wollte die Gruppe sich schnell zur nächsten Station begeben. Ich entschloss mich dazu, näher an den Tisch zu gehen und formte aus einer kleinen Knetkugel eine Schale und legte sie aufs Wasser. Die Schüler sahen $z u$. Trotz, dass sich die Schale nur wenige Sekunden über Wasser halten konnte (sie war etwas löchrig), waren die Schüler sichtlich erstaunt. Danach entfernte ich mich kommentarlos von der Station. Die Schüler entschieden sich dazu, die Station doch nicht zu wechseln und versuchten die Knete zum Schwimmen zu bringen, indem sie die Knete in unterschiedlichste Formen brachten."

Über ihre Rolle als Lernbegleitung schrieb Nadine:

„[...] nahm ich während dieses Termins eher die Rolle als Schülerin ein, indem ich genauso interessiert wie die Kinder beobachtete und eigene Ideen zur Weiterentwicklung des Experiments beitrug. Hierbei nahm ich mich bewusst zurück und interagierte nur, wenn die Kinder in ihrem Gedankengang stockten. Diese Herangehensweise ermöglichte es, neue Erkenntnisse aus einer anderen Perspektive zu gewinnen. Die Kinder nahmen mich auch weniger als Lehrperson, der sie Fragen stellten, sondern mehr als Freundin, der sie ihre Ideen zeigten oder mit der sie Ideen entwickelten und Vermutungen aufstellten, wahr [...]. Zwar war das Lernklima durch meine Position als Schülerin aufgelockert [...]. Doch nach genauerer Reflexion wurde mir bewusst, dass ich trotz der Position sehr stark richtungsweisende Vorschläge einbrachte, die nicht immer an die Ideen anknüpften, so dass ich die Kinder in der Weiterentwicklung ibrer Ideen stoppte oder davon abbrachte."

Die erste (noch recht unstrukturierte) Auswertung der Fragebögen und Aussagen der Studierenden lassen erkennen, dass das Einnehmen der unterschiedlichen Rollen - in erster Linie als Lernbegleitung und als Beobachtende - überwiegend als Chance für den eigenen Lernprozess und für die persönliche Entwicklung bezogen auf die Rolle der Lernbegleitung gesehen wurden. Insbesondere der Erfahrungsaustausch mit den Mitstudierenden, die gemeinsame Reflexion und das gemeinsame Finden von Lösungen für aufgetretene Probleme im Rahmen eines kooperativen Lernprozesses werden als hilfreich eingeschätzt.

In einem nächsten Schritt werden die Fragebögen erweitert, um tiefergehende Einsichten zu erlangen, was die unterschiedlichen Rollen, der Erfahrungsaustausch und die gemeinsame Reflexion im Detail fördern. Darüber hinaus sollen die Studierenden zukünftig standardmäßig Portfolios anfertigen, woraus wir uns erhoffen, Schlüsse über die individuellen Lernprozesse der Studierenden ziehen zu können. 


\section{Fazit}

Das Format des Gorex_Projektpraktikums scheint die Studierenden zum Reflektieren anzuregen. Zusammenfassend bietet das Gofex_PP in seiner Konzeption den Studierenden die Gelegenheit, sich praxisnah in einer Realsituation mit Schüler*innen zu erproben und ergänzend einen „geschützten Rahmen“, in dem diese Erfahrungen, Handlungsschritte, Lernwege und Erkenntnisse (gemeinsam) evaluiert und reflektiert werden. Begründet durch die Einnahme verschiedener Rollen/Perspektiven können die Studierenden in Interaktion gemeinsam und im wechselseitigen Austausch, Kenntnisse und Fertigkeiten erwerben (Konrad \& Traub 2007, 5). Die Weiterentwicklung des Formats Gofex_PP sowie die weitere Erforschung der Einflüsse der Reflexion und des Perspektivenwechsels in den verschiedenen Rollen auf das eigene Lehreverhalten werden Zielsetzung der nächsten Seminare sein.

\section{Literatur}

FSA (Fachspezifischer Anhang zur Prüfungsordnung und zur Studienordnung der Studienfächer der Primarstufe) (2018), Saarbrücken, Universität des Saarlandes, 484-509.

KMK 2017: Qualifikationsrahmen für deutsche Hochschulabschlüsse.

Hagstedt, Herbert (2014): Lernbegleitung - Herausforderung für die Lehrerbildung von morgen. In: Hagstedt, Herbert \& Krauth, Ilse Marie: Lernwerkstätten - Potenziale für Schulen von morgen. Frankfurt am Main, Grundschulverband, 220-231.

Kelkel, Mareike \& Peschel, Markus (2018): Fachlichkeit in Lernwerkstätten. Berücksichtigung von fachlichen Grundlagen beim pädagogischen Handeln in Lernwerkstätten als Chance der Erweiterung bisheriger Lernwerkstätten-Konzeptionen. In: Peschel, Markus \& Kelkel, Mareike (Hrsg.): Fachlichkeit in Lernwerkstätten. Kind und Sache in Lernwerkstätten. Bad Heilbrunn, Klinkhardt, 15-34.

Konrad, Klaus \& Traub, Silke (2007): Kooperatives Lernen in der Schule, Hochschule und Erwachsenenbildung. 2.Auflage. Baltmannsweiler: Schneider Verlag Hohengehren.

Martschinke, Sabine \& Kopp, Bärbel (2015): Kooperatives Lernen. In: Kahlert, Joachim; Fölling-Albers, Maria; Götz, Margarete; Hartinger, Andreas; Miller, Susanne \& Wittowske, Steffen (Hrsg.): Handbuch Didaktik des Sachunterrichts. 2. Auflage. Bad Heilbrunn: Klinkhardt, 424-428.

Peschel, Falko (2012): Offener Unterricht - Idee, Realität, Perspektive. Teil I: Allgemeindidaktische Überlegungen. Teil II: Fachdidaktische Überlegungen. Baltmannsweiler: Schneider Verlag Hohengehren.

Peschel, Markus (2009): Grundschullabor für Offenes Experimentieren - Grundlegende Konzeption. In: Lauterbach, Roland; Giest, Hartmut \& Marquardt-Mau, Brunhilde (Hrsg.): Lernen und kindliche Entwicklung. Bad Heilbrunn: Klinkhardt, 229-236.

Peschel, Markus (2016): Offenes Experimentieren - Individuelles Lernen. Aufgaben in Lernwerkstätten. In: Hahn, Heike; Esslinger-Hinz, Ilona \& Panagiotopoulou, Argyro (Hrsg.): Paradigmen und Paradigmenwechsel in der Grundschulpädagogik. Baltmannsweiler: Schneider Verlag Hohengehren, 120-129.

Renkl, Alexander (2007): Kooperatives Lernen. In: Schneider, Wolfgang \& Hasselhorn, Marcus (Hrsg.): Handbuch Psychologie, Bd. Pädagogische Psychologie. Göttingen: Hogreve, 84-94.

Studienordnung der Universität des Saarlandes für die Studiengänge Lehramt an beruflichen Schulen (LAB), Lehramt für die Primarstufe (LP), Lehramt für Sekundarstufe I (LS1) und Lehramt für 
die Sekundarstufe I und für die Sekundarstufe II (LS1+2) (2018). https://www.math.uni-sb.de/ lehramt/images/pdf/db18_326_studo.pdf [letzter Zugriff am 12.06.2018].

Verbund europäischer Lernwerkstätten (VeLW) e.V. (Hrsg.) (2009): Positionspapier des Verbundes europäischer Lernwerkstätten (VeLW) e.V. zu Qualitätsmerkmalen von Lernwerkstätten und Lernwerkstattarbeit. http://www.forschendes-lernen.net/files/eightytwenty/materialien/VeLW-Broschuere [letzter Zugriff am 12. 06.2018].

Wedekind, Hartmut \& Schmude, Corinna (2017): Werkstätten an Hochschulen - Orte des entdeckenden und/oder forschenden Lernens. In: Kekeritz, Mirja; Graf, Ulrike; Brenne, Andreas; Fiegert, Minika; Gläser, Eva \& Kunze, Ingrid (Hrsg.): Lernwerkstattarbeit als Prinzip. Möglichkeiten für Lehre und Forschung. Bad Heilbrunn: Klinkhardt, 185-200. 